\title{
NUMERICAL STUDY OF AN OIL-WATER FLOW IN A GRAVITATIONAL SEPARATOR
}

\author{
FEDERICO TORRIANO ${ }^{1}$, MARIE-CLAUDE LESSARD ${ }^{1}$, NATHALIE THIBEAULT ${ }^{2}$ \\ ${ }^{1}$ Institut de Recherche d'Hydro-Québec, Varennes, Canada. \\ ${ }^{2}$ Direction expertise DPPEAR d'Hydro-Québec, Montréal, Canada.
}

\begin{abstract}
During their operation or in the event of an accident, power transformer can release a certain amount of oil in the subjacent soil. In order to avoid a fire hazard or any contamination to the environment, it is critical to capture any oil that was accidentally spilled. For this reason, catchment basins are placed below each power transformer and each basin is connected through pipes to a gravitational oil-water separator, which allows the oil droplets carried by the water flow to rise toward the surface and coalesce near the free surface. By doing so, the oil phase is separated from the mixture and it can be properly disposed afterwards. Prior to 1995, gravitational separators at Hydro-Québec have not been designed according to the American Petroleum Institute (API) standards [1] but this does not necessarily imply that such separators do not comply with the environmental legislation in place. Thus, in order to evaluate if modifications to the existing gravitational separators are required, Hydro-Québec has launched in 2012 an R\&D project aimed at performing separator efficiency studies through a Computational Fluid Dynamics (CFD). In this paper, a numerical simulation of a gravitational oil-water separator in service at Hydro-Québec using an inhomogeneous multiphase model is presented. Moreover, a new configuration of the existing separator is numerically tested and the results show that its performance is significantly improved. Keywords: CFD, inhomogeneous model, oil-water separator, two-phase flow
\end{abstract}

\section{INTRODUCTION}

Hydro-Québec is a publicly owned company among the largest electric utilities in Canada that generates, transmits and distributes electricity. The transmission division of HydroQuébec has the most extensive network in North America with $34000 \mathrm{~km}$ of power lines and more than 500 substations. Each substation is usually equipped with several power transformers that can contain up to 500001 of naphthenic mineral oil each to ensure their cooling. Thus, during their operation or in the event of an accident, power transformers can release a certain amount of oil in the subjacent soil and in order to avoid a fire hazard or any contamination to the environment, it is critical to capture any oil that could be accidentally spilled. For this reason, as shown in Fig. 1, catchment basins are placed below each power transformer and each basin is connected, through pipes, to a gravitational oil-water separator. The latter device generally consists of a large rectangular concrete tank filled with water and equipped with a free-surface inlet on one side and a submerged outlet on the opposite side. The objective of the gravitational separator is to allow the oil droplets carried by the water flow to rise toward the surface and coalesce near the free surface. By doing so, the oil phase is separated from the mixture and it can be properly disposed afterwards.

Prior to 1995, gravitational separators at Hydro-Québec have not been designed according to the American Petroleum Institute (API) standards [1] (which are based on Stokes' law for spherical droplets) but this does not necessarily imply that such separators do not comply with the environmental legislation in place. Thus, in order to evaluate if existing gravitational separators need to be modified, Hydro-Québec has launched in 2012 an R\&D project aimed at performing separator efficiency studies through a Computational Fluid Dynamics (CFD) approach. Furthermore, if modifications to the existing design are required, the new separator 


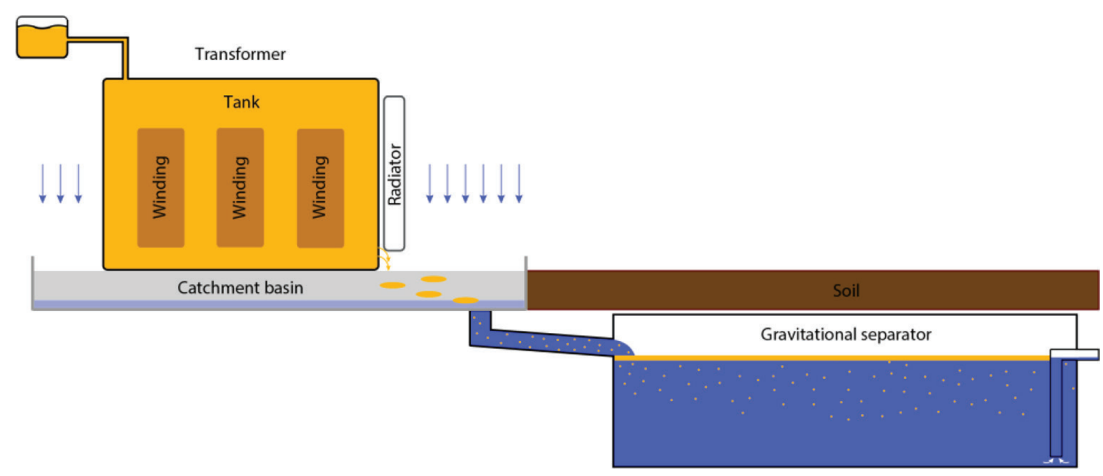

Figure 1: Schema of catchment basin and gravitational separator in a substation.

configuration will be tested through CFD in order to optimize the separator performance while minimizing the implementation costs.

This numerical approach has been used in the past to investigate the fluid flow in gravitational separators. For example, Hansen et al. [2] have performed simulations of a water-oil flow in a separator using an in-house code and have shown that the model predictions were in quite good agreement with the experimental data. Moreover, the simulations have allowed testing different inlet configurations and various operating conditions. A later study performed by the same author [3] using a commercial software has also shown that CFD modelling was a valuable tool in the design or redesign of gravity separators. In Holdo and Calay [4], a steady-state CFD simulation of an offshore oil-water gravity separator is done using an interphase slip algorithm model. The results have shown that the oil droplets size and inlet flow velocity have a significant impact on the separation efficiency. The same conclusions have been reached in the numerical investigation carried out by Abdulkadir et al. [5]. Furthermore, Behin and Aghajari [6] have performed a numerical and experimental analysis of an offshore oil-water separator and have shown that an increase of the water level in the vessel tends to decrease and increase the mean residence time of the organic and aqueous phases respectively. In the experimental study carried out by Das and Biswas [7], the efficiency of baffles in oil-water separation has been tested and it has been found that, for the tested operating conditions, a $45^{\circ}$ baffle inclination to vertical provided the best separation performance. Another study performed by Wilkinson et al. [8] on baffle plate configurations has shown that two baffles are in general more beneficial to phase separation than a single baffle if they are very closely spaced.

In this paper, a CFD simulation of a gravitational oil-water separator in service at HydroQuébec is performed using an inhomogeneous multiphase model and a detailed analysis of the flow dynamics is provided. Moreover, a new configuration of the existing separator is numerically tested and its performance is compared with the one of the original design.

\section{SEPARATOR GEOMETRY AND OPERATING CONDITIONS}

As shown in Fig. 2, the gravitational separator has a hexahedral shape except near the bottom where the side walls have a $45^{\circ}$ slope. Two inlets are present: the main inlet (inlet 1 ) is centered with respect to the separator width whereas the secondary inlet (inlet 2) is placed on the side of the separator at a $90^{\circ}$ angle with respect to the main inlet. The outlet is positioned in front of the main inlet at a distance of $0.2 \mathrm{~m}$ from the back wall of the separator. The total volume of the basin is $175 \mathrm{~m}^{3}$. 

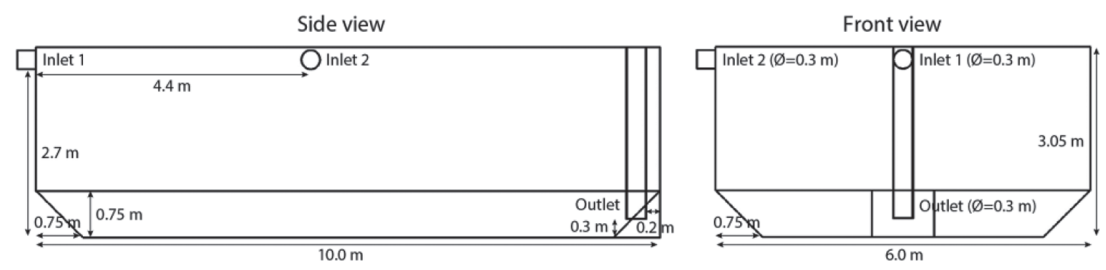

Figure 2: Separator geometry.

In order to simulate a realistic scenario, the water flow rates at the two inlets are computed from the IDF (rainfall intensity, duration and frequency) curves at the substation site and from the surface of the catchment basin. The oil flow rates are computed from the amount of oil that could be spilled due to a transformer failure. Thus, at the main inlet a water mass flow rate of $29 \mathrm{~kg} / \mathrm{s}$ and an oil mass flow rate of $0.248 \mathrm{~kg} / \mathrm{s}$ are specified whereas at the secondary inlet a water mass flow rate of $5 \mathrm{~kg} / \mathrm{s}$ and an oil mass flow rate of $0.043 \mathrm{~kg} / \mathrm{s}$ are set. This fluid phase distribution corresponds to inlets oil concentrations of almost $10000 \mathrm{ppm}$. As the oil is expected to be carried to the separator by the water flow under the form of small droplets (i.e. dispersed phase), the size distribution of such droplets needs to be known. Unfortunately, no such measurements have yet been performed for in-situ gravitational separators and no information is currently available in the literature. Moreover, the droplets size distribution will depend, among other factors, on the catchment basin geometry and gravel bed layout, the water and oil flow rates, and the piping configuration between the catchment basin and the separator. Thus, a theoretical size distribution of $1 \%$ and $99 \%$ volume fraction of $100 \mu \mathrm{m}$ and $2000 \mu \mathrm{m}$ droplets diameters respectively is assumed. These two oil droplets diameters have been chosen in order to reproduce the expected minimum and maximum sizes in a real gravitational separator. The fluid properties of the continuous phase (water) and dispersed phase (oil) given below are evaluated at $5^{\circ} \mathrm{C}$ (i.e. autumn and spring scenarios) in order to be conservative since at this temperature the density difference between the water and oil is lower than at $25^{\circ} \mathrm{C}$ (i.e. summer scenario):

$$
\begin{gathered}
\rho_{\text {water }}=1000\left[\frac{\mathbf{k g}}{\mathbf{m}^{3}}\right] \\
\mu_{\text {water }}=0.00115[\mathrm{~Pa} \cdot \mathrm{s}] \\
\rho_{\text {oil }}=877.5\left[\frac{\mathbf{k g}}{\mathbf{m}^{3}}\right] \\
\mu_{\text {oil }}=0.0197[\mathrm{~Pa} \cdot \mathrm{s}]
\end{gathered}
$$

\section{NUMERICAL MODEL}

The numerical results presented in the current paper have been obtained with the commercial code Ansys-CFX v16.0. This software is a finite volume type solver and it uses the integral form of the conservation and transport equations (i.e. Navier-Stokes equations) to solve all variable of interest. Thus each variable $(\varphi)$ is solved at the center of the control volume (CV). In order to evaluate the convective and diffusive fluxes at the surfaces of the $\mathrm{CV}$, a quasisecond order spatial interpolation scheme (i.e. 'high resolution') and a second-order 
('backward Euler') temporal interpolation scheme are used. The convergence criterion on the rms (root mean square) residual values is set at $1 \mathrm{e}-6$.

Due the flow velocity at the inlet of the separator, the jet is turbulent (i.e. $\operatorname{Re}_{\text {inlet }}>18000$ ), and thus, a turbulence model is required. In the current case the $\mathrm{k}-\varepsilon$ model with a wall function approach has been selected.

Furthermore, to solve the diphase flow, an inhomogeneous model is used and the NavierStokes equations are solved for each phase as follows:

$$
\begin{gathered}
\frac{\partial}{\partial t}\left(r_{\alpha} \rho_{\alpha}\right)+\nabla \cdot\left(r_{\alpha} \rho_{\alpha} \boldsymbol{U}_{\alpha}\right)=0 \\
\frac{\partial}{\partial t}\left(r_{\alpha} \rho_{\alpha} \boldsymbol{U}_{\alpha}\right)+\nabla \cdot\left(r_{\alpha}\left(\rho_{\alpha} \boldsymbol{U}_{\alpha} \times \boldsymbol{U}_{\alpha}\right)\right)=-r_{\alpha} \nabla p_{\alpha} \\
+\nabla \cdot\left(r_{\alpha} \mu_{\alpha}\left(\nabla \boldsymbol{U}_{\alpha}+\left(\nabla \boldsymbol{U}_{\alpha}\right)^{T}\right)\right)+M_{\alpha},
\end{gathered}
$$

where $r_{\alpha}$ is the volume fraction of phase $\alpha$ and $M_{\alpha}$ is the interfacial force term between the phases. In the present case, only the drag force is considered as an active interfacial force and the Shiller-Naumann model is employed since the concentration of the oil droplets is quite low (i.e. $r_{\text {oil }}<10000 \mathrm{ppm}$ ). It should be mentioned that the numerical model employed in this study does not take into account the oil droplets coalescence and fragmentation phenomena.

\subsection{Domain discretization}

As illustrated in Fig. 3, the computational domain is discretized using a hexahedral mesh. The grid is more refined near the two inlets of the separator in order to better capture the jets dynamics as well as close to the separator walls in order to have an average $\mathrm{y}^{+}$value close to 30 . The overall mesh size is $2.56 \mathrm{M}$ elements.

\subsection{Boundary conditions}

At the two separator inlets, the water and oil mass flow rates described in Section 2 are specified and at the outlet a uniform pressure condition $(\mathrm{p}=0 \mathrm{~Pa})$ is set. On all the separator walls

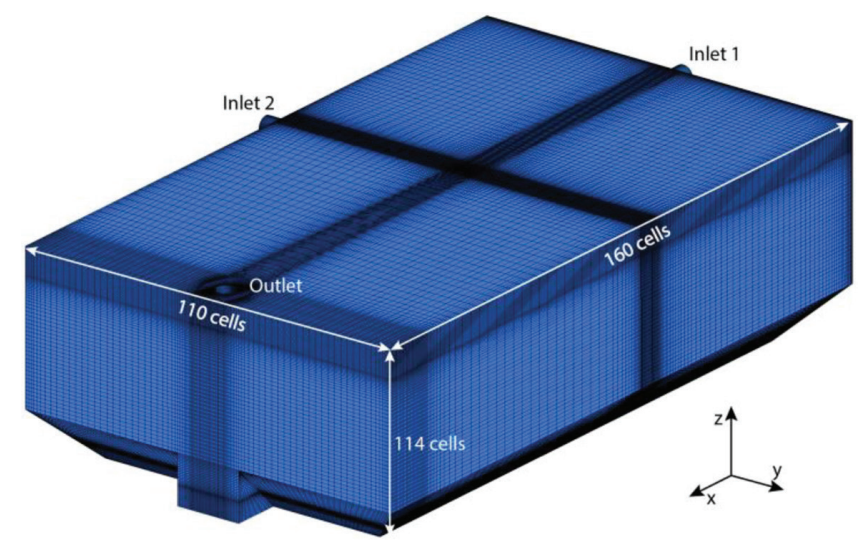

Figure 3: Domain discretization. 
a no-slip condition is imposed except for the top surface of the basin where a free-slip condition is specified in order to reproduce the free surface. At $t=0 \mathrm{~s}$, the separator is filled with stagnant water and no oil layer is present at the surface. The simulation time step is $0.025 \mathrm{~s}$ for the first two minutes of physical time (i.e. Courant number of 0.4 ), $0.05 \mathrm{~s}$ for the following three minutes and $0.1 \mathrm{~s}$ for the remaining time. The total simulation time is 60 minutes and the computation duration on 128 parallel processors was about two weeks.

\section{RESULTS}

This section of the paper is subdivided in two parts: in the first one, the numerical results of the original separator design are analyzed and in the second one the performance of a new separator design is investigated.

In the graph of Fig. 4, the isosurface of $100 \mathrm{ppm}$ of oil concentration (for $100 \mu \mathrm{m}$ diameter droplets) is shown in yellow whereas the contours of oil concentration are plotted on an $x-y$ plane located at $0.25 \mathrm{~m}$ from the separator bottom and on an $\mathrm{x}-\mathrm{z}$ plane centered on the width of the separator.

At first, one can notice the interaction between the primary and secondary inlet jets. Due to the much higher mass flow rate of the primary flow, the secondary flow cannot reach the separator wall located in front of inlet 2 and it is instead deflected toward the x-direction. Thus, the two inlet flows tend to merge together after about $70 \mathrm{~s}$ and the time required for the $100 \mu \mathrm{m}$ oil droplets to cross the entire length of the separator is about $105 \mathrm{~s}$. Afterwards, the jet impacts with the separator back wall and it is deflected toward the bottom. The first 100 $\mu \mathrm{m}$ oil droplets reach the separator outlet about $150 \mathrm{~s}$ after the beginning of the spill and this time period is much lower than the theoretical mean residence time (i.e., $86 \mathrm{~min}$ ). The larger oil droplets (i.e. $2000 \mu \mathrm{m}$ diameter) never reach the separator outlet and they remain at the surface of the separator.

A better evaluation of the separator performance can be made from the plot of Fig. 5, where the oil concentration at the separator outlet is plotted as a function of time. Again one can notice that the first oil front reaches the separator outlet $150 \mathrm{~s}$ after the beginning of the spill and it causes an oil leakage of $30 \mathrm{ppm}$ concentration for a period of about $20 \mathrm{~s}$. Afterward, the outlet oil concentration rapidly decreases to $22 \mathrm{ppm}$ and then steadily increases up
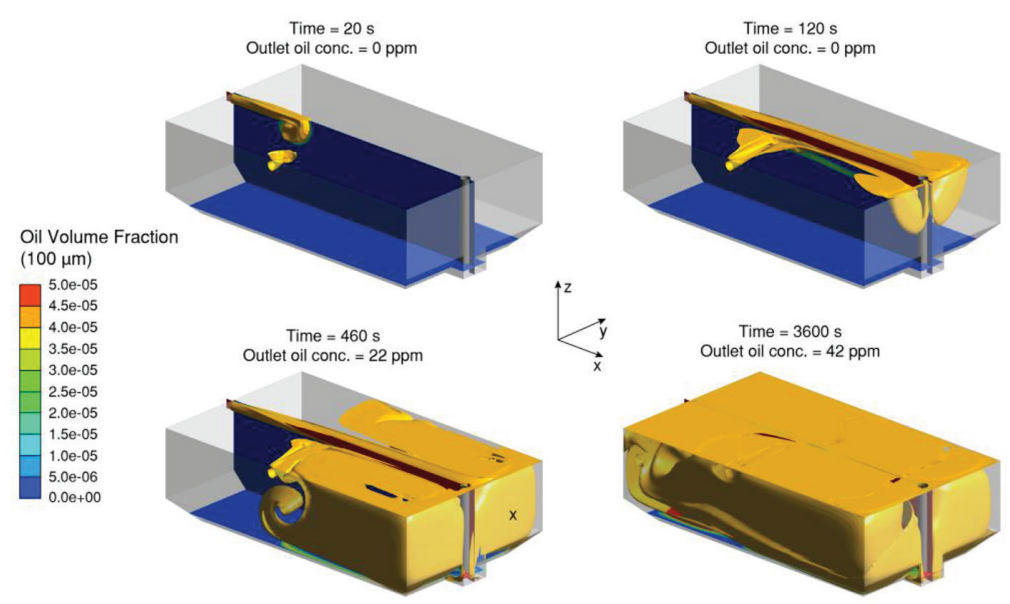

Figure 4: Temporal evolution of oil concentration in separator. 


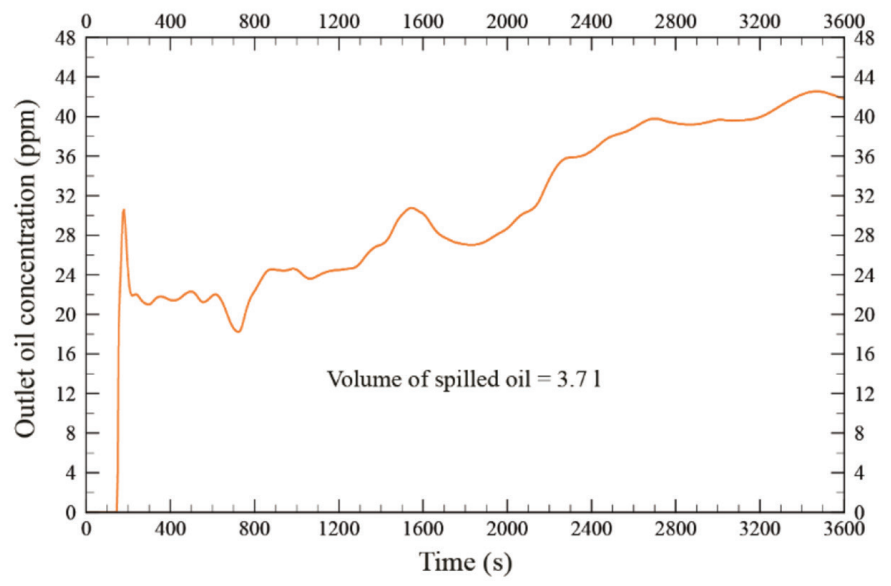

Figure 5: Temporal evolution of oil concentration at separator outlet.

to a value of $42 \mathrm{ppm}$ one hour after the spill started. This progressive increase of the outlet oil concentration could be in part due to the fact that the numerical model cannot account for the coalescence phenomenon and consequently, even if the oil droplets reach a high concentration near the separator free surface, they do not form a layer and in principle they can be entrained again by the flow toward the separator outlet.

The CFD results have shown that the current separator configuration is not optimal since the outlet is located directly in front of the main flow inlet and consequently the smallest oil droplets can rapidly reach the exit. Thus, to improve the separator performance, it would be recommended to locate the outlet much closer to the main inlet in order to delay the arrival of the first oil front and to increase the residence time of the oil droplets in the separator. This new design is tested in the following section.

\subsection{Modified separator design}

As discussed in the previous section, the original separator design does not seem optimized mainly due to the outlet location. Thus, a new numerical study was done in order to find a configuration that would improve the separator performance while reducing the time and cost associated with the in-situ implementation of such modifications. Based on the analysis of the flow dynamics in the original separator, it was found that the best location for the outlet would be at a downstream distance of $2 \mathrm{~m}$ from the main inlet and a 2-m distance from the side wall, as illustrated in Fig. 6.
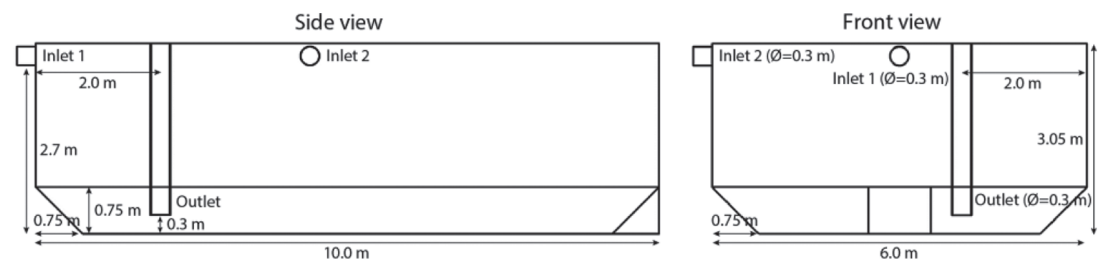

Figure 6: Modified separator geometry. 


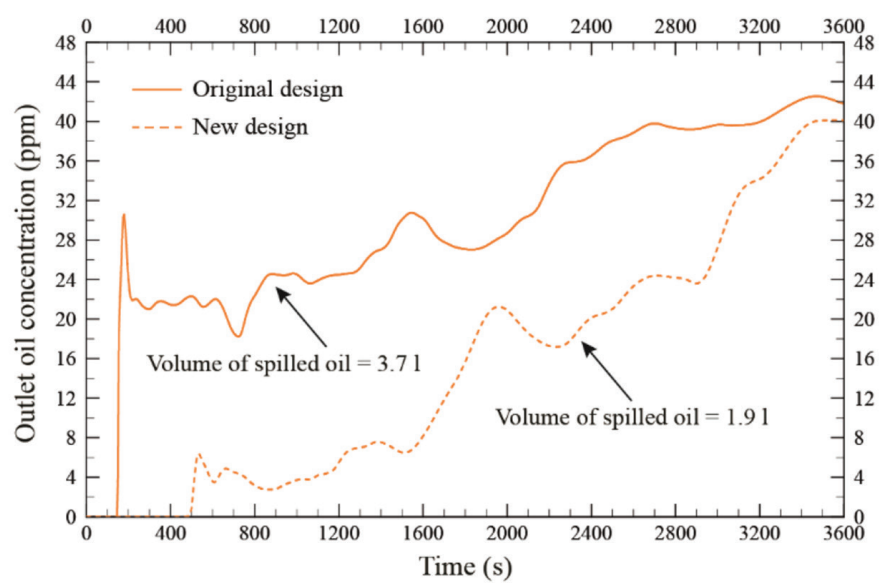

Figure 7: Oil concentration at outlet of original and new separator designs.

In order to validate the proposed design, a new CFD simulation was run using the same numerical parameters and boundary conditions of the previous computation. The plots of Fig. 7 show the comparison between the temporal evolutions of the outlet oil concentration for the original and the modified separator designs. Firstly, it is possible to notice that, with the new design, the arrival of the oil front is delayed by almost 6 min. Moreover, the intensity of the oil front is greatly reduced (i.e. $6 \mathrm{ppm}$ instead of $30 \mathrm{ppm}$ ). In the second place, during the initial $27 \mathrm{~min}$ of the oil spill, the outlet oil concentration in the modified separator is about $20 \mathrm{ppm}$ lower than the one computed for the original design, and even for the remaining simulated time it remains at an inferior value. Consequently, the total amount of oil that is spilled during the one hour period is 1.91 whereas it was 3.71 in the original separator.

In summary, the numerical approach used in the present study has allowed to successfully analyze the diphase flow in a separator in service at Hydro-Québec at to propose a new design that significantly improves the separation performance of the equipment. Moreover, it was found than placing the outlet directly in front of an inlet could be detrimental to the separator performance since the inlet jet is deflected toward the bottom of the separator (and thus toward the outlet intake) by the back wall of the basin.

\section{CONCLUSION}

In the current study, a 3D diphase transient simulation of the flow in a gravitational separator in service at Hydro-Québec has been performed. The numerical results show that the oil front reaches the separator outlet quite rapidly due to the location of the outlet, which is in front of the main inlet. Based on a flow dynamics analysis, a new separator design has been proposed and tested through numerical modeling. In the modified configuration, the outlet is located closer to the main inlet in order to delay the arrival of the oil front at the separator exit and to allow the oil droplets to rise toward the free surface. According to the numerical results, the oil concentration at the outlet of the modified separator is significantly decreased and the total amount of spilled oil is reduced by half compared to the original configuration.

CFD has proven to be a valuable tool in the analysis and improvement of gravitational separator performance since it provides very detailed information on the flow dynamics inside the separator. In the future, a special effort will be put in determining the oil droplets 
diameter distribution at the inlet of existing separators since this parameter is known to have a great effect on the separator performance. Moreover, coalescence and fragmentation phenomena will also be included in the model.

\section{REFERENCES}

[1] Monographs on Refinery Environmental Control-Management of Water Discharges, Design and Operation of Oil-Water Separators. American Petroleum Institute Publication 421, 1990.

[2] Hansen, E.W.M., Heitmann, H., Lakså, B., Ellingsen, A., Østby, O., Morrow, T.B. \& Dodge, F.T., Fluid flow modelling gravity separators. Proceedings of the 5th International Conference on Multi-Phase Production, Cannes, France, 1991.

[3] Hansen, E.W.M., Phenomenogical modelling and simulation of fluid flow and separation behaviour in offshore gravity separators. ASME Pressure Vessels and Piping Division, 431, pp. 23-29, 2001.

[4] Holdo, A.E. \& Calay, R.K., Two-phase flow modelling for industrial applications. Fluid Mechanics Research Group, University of Hertfordshire, UK, 2006.

[5] Abdulkadir, M., Hernandez-Perez, V. \& Hossain, M., Modelling of oil-water separator using computational fluid dynamics (CFD). Proceedings of the 7th International Conference on Heat Transfer, Fluid Mechanics and Thermodynamics, Antalya, Turkey, 2010.

[6] Behin, J. \& Aghajari, M., Influence of water level on oil-water separation by residence time distribution curves investigations. Separation and Purification Technology, 64, pp. 48-55, 2008.

https://doi.org/10.1016/j.seppur.2008.08.009

[7] Das, S.K. \& Biswas, M.N., Separation of oil water mixture in tank. Chemical Engineering Communications, 190, pp. 116-127, 2003.

https://doi.org/10.1080/00986440302095

[8] Wilkinson, D., Waldie B., Nor, M.I.M. \& Lee, H.Y., Baffle plate configurations to enhance separation in horizontal primary separators. Chemical Engineering, 77, pp. 221-226, 2000.

https://doi.org/10.1016/s1385-8947(99)00170-9 